\title{
Analysis of sperm quality in recombinant inbred mouse strains: correlation of sperm head shape with sperm abnormalities and with the incidence of supplementary spermatozoa in the perivitelline space
}

\author{
H. Krzanowska, J. Styrna and B. Wabik-Śliz \\ Department of Genetics and Evolution, Institute of Zoology, Jagiellonian University, R. Ingardena 6, \\ 30-060 Kraków, Poland
}

\begin{abstract}
Recombinant inbred strains were developed from reciprocal crosses between two inbred strains of mice (CBA and $K E$ ) differing in sperm head shape, proportion of normal sperm heads (CBA, 95\%; KE, 78\%) and fertilization efficiency (CBA, 100\% of fertilized ova; KE, $72 \%)$, to determine whether the indices of sperm morphology and function were correlated. The following parameters were analysed in recombinant inbred and progenitor strains: index of sperm head shape (head width in the middle of its length/head length), percentage of abnormal sperm heads, percentage of spermatozoa with progressive movements, efficiency of penetration of hyaluronic acid polymer (Sperm Select ${ }^{R}$ ) and percentage of fertilized ova after mating males from the tested strains with females from an outbred stock. For each investigated character, recombinant inbred strains, recombinant inbred EXCB and CBXE, could be divided into at least three categories: KE-like, CBA-like and intermediate, suggesting that in each case a minimum of two genes was involved. Recombinant strains derived from the reciprocal crosses of progenitor strains differed only with respect to the proportion of abnormal sperm heads, showing the involvement of the $Y$ chromosome in determining this character. Penetration into Sperm Select was significantly correlated both with fertilization efficiency and sperm motility, while correlation with the proportion of normal spermatozoa did not reach the level of significance. However, there was a significant negative correlation of both sperm abnormalities and the incidence of supplementary spermatozoa in the perivitelline space with the index of sperm head shape. The results indicate that these characters are genetically linked, or that sperm heads with a lower width to length ratio are more prone to undergo deformations during spermiogenesis, and are less efficient in establishing contact with the vitellus after penetrating the zona pellucida.
\end{abstract}

\section{Introduction}

The successful spermatozoon must possess specific morphological and physiological features to be able to get into the vicinity of the egg and fertilize it. The various components of sperm morphology and function are usually correlated, for example spermatozoa of men with lowered fertility tend to have low concentration, a high proportion of abnormalities, lower motility and ability of penetrating the cervical mucus, binding to the zona pellucida and penetrating the egg (see Aitken et al., 1992; Liu and Baker, 1992; and Kaskar et al., 1994; and the literature cited therein). It is possible that disturbances of the process of spermatogenesis may simultaneously affect many aspects of sperm performance. However, the relationship between sperm morphology and function was described by Liu and Baker (1992): studying the morphology of spermatozoa bound to the zona pellucida, they found that both sperm head shape and normality were highly correlated with fertilization

Received 6 March 1995 rates in vitro, although there were exceptions. The relationship between sperm morphology and function can be investigated more thoroughly in studies using males with specific features of sperm morphology. Inbred strains differing in sperm quality owing to genetic factors are therefore suitable models.

Two strains of mice, KE and CBA, differ with respect to sperm head shape (Krzanowska, 1986) and function: KE males are characterized by a higher proportion of abnormal sperm heads (Krzanowska, 1969, 1976) and lower fertilization rate, both in vivo (Krzanowska, 1970) and in vitro (Kaleta, 1977). Recombinant inbred strains were developed from reciprocal crosses between $\mathrm{KE}$ and $\mathrm{CBA}$ strains, to determine whether the indices of sperm morphology and function, which were correlated in the progenitor strains, would segregate jointly. Recombinant inbred strains, first developed by Bailey (1971), are especially useful for studying genetic and physiological correlations between characters, because each recombinant inbred strain provides a repeatable, homozygous sample of particular recombination (haplotype) of alleles that were different in the progenitor strains. In this study, the 
following parameters related to sperm morphology and function were estimated among the progenitor and RI strains: sperm head shape and abnormalities, sperm motility, penetration into hyaluronic acid polymer Sperm Select ${ }^{R}$, and efficiency of fertilization in vivo. It was assumed that significant correlations should point to parameters that are genetically or physiologically linked with each other.

\section{Materials and Methods}

\section{Mice}

Adult male mice (3-7 months old) from the following inbred strains were used: $\mathrm{CBA} / \mathrm{Kw}$ (referred to as $\mathrm{CBA}$ ), $\mathrm{KE}$, and recombinant inbred strains developed in our Department: recombinant inbred EXCB 1, 2, 3, 5, 6, 7, 10 obtained from the original cross of $\mathrm{KE}$ females with $\mathrm{CBA}$ males, and recombinant inbred CBXE 12, 13, 14, 15, 17, 18, 20 from the reciprocal cross. Unfortunately, five recombinant inbred strains $(1,3,7,14$ and 18) were lost before the end of experiments, and could not be used to evaluate penetration into Sperm Select and fertilization. Incipient recombinant inbred CBXE 21-34 strains were also included so that sperm shape and abnormalities could be estimated; these strains have passed through 12-14 generations of inbreeding. All mice were bred in our Department and were given the commercial pelleted diet (Murigran, ZDP Lublin, Poland); lights were switched on between 09:00 $\mathrm{h}$ and 21:00 h. Male mice were caged singly for at least 3 days before they were used in experiments.

\section{Sperm head morphology}

Spermatozoa expressed from the vas deferens were dispersed in physiological saline, smeared on slides, fixed in acetic alcohol and stained with Eosin Y (Wako Pure Chemical Industries, Osaka); the proportion of abnormal sperm heads was calculated from 200 spermatozoa for each male (Krzanowska, 1976). The morphology of normal sperm heads was analysed on the photographs, by calculating a simple index of sperm head shape, according to the formula $200 \times W / L$, where $W$ is sperm head width measured in the middle of its length, and $L$ is the length of sperm head; 30 sperm heads (from two males) were measured for each strain.

\section{Sperm Select penetration test}

After killing the male mouse by cervical dislocation, each cauda epididymis was gently squeezed with forceps and the content of each vas deferens was expressed into $50 \mu \mathrm{M} \mathrm{M} 2$ medium (Sigma, St Louis, MO) containing 2\% (w/v) bovine albumin fraction $V$ (Sigma), allowing spermatozoa to disperse for $5 \mathrm{~min}$. A small sample was used to count the number of spermatozoa and those with progressive movements in a haemocytometer. Sperm Select penetration test was performed as described by Aitken et al. (1992) for human spermatozoa, with some modifications. Sperm Select ${ }^{1}$. (Pharmacia AB, Uppsala, Sweden) was diluted 1:1 with M2 medium and loaded into capillary tubes (internal diameter $0.95 \mathrm{~mm}$, length $5.0 \mathrm{~cm}$ ).
One end of each tube was sealed in a flame, whereas the other end was inserted into a $25 \mu \mathrm{l}$ aliquot of sperm suspension and incubated in a vertical position for $30 \mathrm{~min}$ at $37^{\circ} \mathrm{C}$. At the end of this period, the tube was removed from the semen and placed on the stage of a compound microscope, equipped with a $\times 10$ objective. Sperm density was assessed at 1.0, 1.5, 2.0, 2.5 and $3.0 \mathrm{~cm}$ from the base of the tube, by counting spermatozoa present in the optical section of one microscope field. The estimated number of spermatozoa was the mean of two independent determinations by two workers. For each male mouse two separate experiments were made (one for each vas deferens) and the better result was always taken for further calculations.

\section{Proportion of fertilized ova and the incidence of supplementary spermatozoa}

Females from an outbred stock were caged individually overnight with experimental males and killed 1 day after mating. At least five males, mated to about 10 females, were used for testing each strain. Ova were flushed from the oviducts and examined for the presence of spermatozoa under the zona pellucida. Two-cell ova with the second polar body and clearly visible nuclei were considered as fertilized. One-cell ova, if present, were dried on slides, fixed in acetic alcohol and stained with toluidine blue to reveal chromatin structures (Krzanowska and Lorenc, 1983); ova at metaphase II of meiosis were considered as unfertilized. Fragmented ova, found only sporadically, were discarded. Ova from sterile copulations were not counted. In one experiment, females were mated with $\mathrm{KE}$ males between 09:00 and 12:00 $\mathrm{h}$ and inspected every $15 \mathrm{~min}$ for the presence of a copulation plug; they were killed by cervical dislocation, $4-5 \mathrm{~h}$ after mating, and their ova were examined as described above.

\section{Statistical analysis}

For statistical treatment, percentages were transformed into angles (Snedecor, 1955). The differences between groups were compared by analysis of variance (ANOVA), unless otherwise indicated.

For each recombinant inbred strain, the value of the given parameter of sperm quality was compared with the respective values for both $\mathrm{KE}$ and $\mathrm{CBA}$ strains; accordingly, the recombinant inbred strains were ascribed to the following phenotypes: I - KE-like, significantly different from CBA but not from $K E ; 2$ - CBA-like, significantly different from $\mathrm{KE}$ but not from CBA; 3 - intermediate, significantly different from both KE and CBA strains; 4 - not different from either parental strain. The number of genes involved in determining the given parameter was determined using the equation from Bailey (1981):

$$
\text { minimum number of effective } \operatorname{loci}=\log (F+2) / \log 2
$$

( $F=$ number of recombinant strain phenotypes different from progenitor phenotypes). 


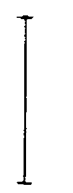

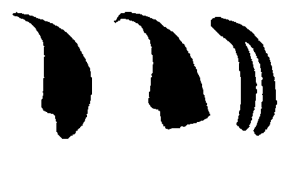

n

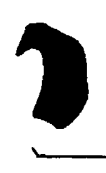

3

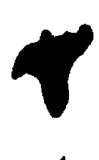

4
Fig. 1. Normal sperm head (n) and types of abnormalities (1-4) in mouse spermatozoa. Scale bar represents $10 \mu \mathrm{m}$.
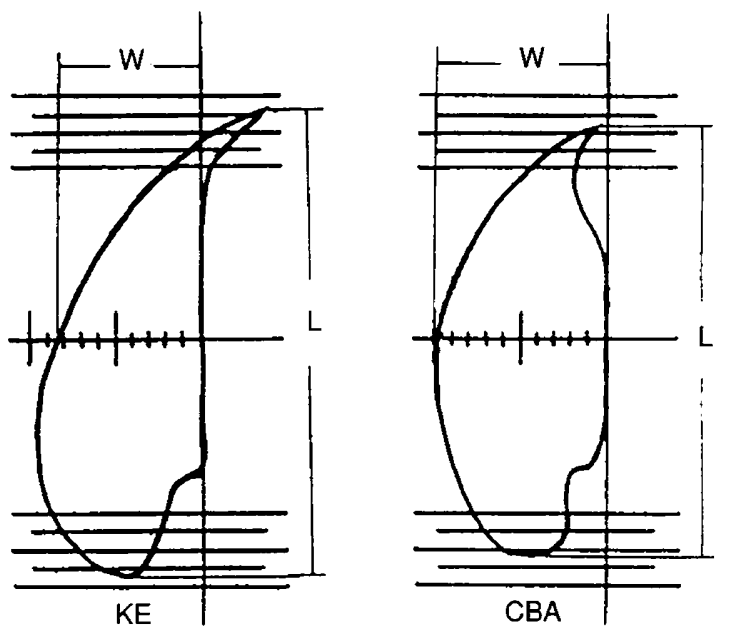

Fig. 2. Normal sperm heads of KE and CBA male mice, with superimposed lines that were used for estimating index of sperm shape; L: head length; $W$ : width measured in the middle of the length.

\section{Results}

\section{Sperm morphology}

The proportion of abnormal sperm heads was significantly higher in the KE than in the CBA strain (Table 1). Among the recombinant inbred strains, there were only two that were not different from $\mathrm{CBA}$ (both belonging to the recombinant inbred EXCB group), 14 intermediate, six not different from KE and two (both belonging to the recombinant inbred CBXE group) with a significantly higher proportion of abnormalities than in the KE strain. Thus two phenotypes were different from the phenotypes of progenitor strains, indicating that a minimum of two gene loci was involved. The mean value for all recombinant inbred CBXE strains ( $Y$ chromosome from the KE male which sired the original cross) was significantly higher than for RI EXCB strains ( $Y$ chromosome from CBA), showing the role of the $\mathrm{Y}$ chromosome in determining the level of sperm abnormalities. The abnormality types were similar in both progenitor strains and recombinant inbred strains, types 2 and 3 (Fig. 1) representing $65-85 \%$ of all abnormalities.

As shown in Fig. 2, both progenitor strains differ conspicuously in the shape of normal sperm heads, so that they could easily be distinguished from one another (Krzanowska, 1986). They have different curvature of the dorsal contour: $C B A$ sperm heads are widest in the middle of their length and KE in the basal part; moreover, CBA spermatozoa possess a characteristic invagination in the apical part of the head. The simple index of sperm head shape used in this study was significantly higher for the CBA than for the KE strain (Table 1 ) and allowed recombinant inbred strains to be separated into KE-like (seven strains), CBA-like (one strain) and intermediate (16 strains). However, the intermediate recombinant inbred strains could still be divided into three partially overlapping groups, so that the total number of phenotypes different from phenotypes of progenitor strains amounted to three, suggesting that a minimum of three gene loci were involved. It should be stressed that spermatozoa with an index value below 70 or about 80 could at first glance be recognized as belonging to KE or $\mathrm{CBA}$ type, respectively. The difference between recombinant inbred EXCB and CBXE strains was not significant, suggesting that the $Y$ chromosome does not influence this character.

\section{Motility and Sperm Select penetration}

There were no significant differences between strains in the mean number of spermatozoa expressed from the vasa deferentia. The proportion of spermatozoa showing progressive movements was significantly higher in the CBA than in the KE strain (Table 2), and recombinant inbred strains were divided into four CBA-like, two KE-like and three not different from either strain.

Numbers of spermatozoa measured at successive distances from the base of Sperm Select preparation declined in an exponential manner in relation to penetration depth, from highly variable numbers (sometimes $>100$ ) present at $1 \mathrm{~cm}$ to only a single spermatozoon at $3.0 \mathrm{~cm}$. For each male, linear regression lines were drawn for numbers of spermatozoa in log transformation in relation to penetration depth. The points at which these regression lines bisected the abscissa (cf. Fig. 3) represented the theoretical distance at which sperm density reached zero, or theoretical vanguard distance (TVD) (Aitken et al., 1992). Mean numbers of spermatozoa at $2.5 \mathrm{~cm}$ depth and TVD values for each strain were significantly lower for KE than for CBA spermatozoa, and recombinant inbred strains could be divided into CBA-like, KE-like and not different from either strain (Table 2). One strain (RI 12) showed an even lower value than KE, but the difference was significant for the TVD value only. The difference between recombinant inbred EXCB and $C B X E$ strains was not significant.

\section{Proportion of fertilized ova and the incidence of supplementary spermatozoa}

The efficiency of fertilization was estimated from all eggs analysed after mating males from each strain with females from an outbred stock. The differences in the proportions of fertilized ova were evaluated by the test of independence (chi square, Yates' correction). CBA males showed significantly higher efficiency of fertilization that did the KE males (Table 3). Among the recombinant inbred strains, two were CBA-like, six KE-like and one intermediate. Recombinant inbred EXCB strains did not differ in this respect from recombinant inbred CBXE strains.

Some fertilized as well as unfertilized ova contained one (sometimes two) supplementary spermatozoa under the perivitelline space (Table 3). The proportion of such ova was higher in matings with $\mathrm{KE}$ males than with $\mathrm{CBA}$ males, and the 
Table 1. Proportion of abnormal spermatozoa and indices of sperm shape in the progenitor strains $C B A$ and $\mathrm{KE}$, and in recombinant inbred strains, RI EXCB and CBXE

\begin{tabular}{|c|c|c|c|c|c|}
\hline \multirow[b]{2}{*}{ Strain } & \multirow[b]{2}{*}{$n$} & \multicolumn{2}{|c|}{ Abnormal spermatozoa (angles) } & \multicolumn{2}{|c|}{ Index of sperm head shape ${ }^{a}$} \\
\hline & & Mean \pm SEM & Type $^{b}$ & Mean \pm SEM & Type $^{b}$ \\
\hline $\mathrm{CBA}$ & 20 & \multicolumn{2}{|l|}{$13.45 \pm 0.46$} & \multicolumn{2}{|l|}{$89.54 \pm 1.69$} \\
\hline $\mathrm{KE}$ & 20 & \multicolumn{2}{|l|}{$27.80 \pm 0.95^{c}$} & \multicolumn{2}{|l|}{$65.92 \pm 1.28^{c}$} \\
\hline RI EXCB I & 12 & $20.00 \pm 0.82$ & $x$ & $86.47 \pm 1.54$ & $\mathrm{CB}$ \\
\hline 2 & 10 & $17.60 \pm 0.82$ & $x$ & $81.37 \pm 1.24$ & $x$ \\
\hline 3 & 10 & $13.70 \pm 0.80$ & $\mathrm{CB}$ & $73.80 \pm 1.65$ & $x$ \\
\hline 5 & 10 & $16.00 \pm 1.19$ & $\mathrm{CB}$ & $77.80 \pm 0.96$ & $x$ \\
\hline 6 & 14 & $18.86 \pm 0.54$ & $x$ & $77.90 \pm 1.83$ & $x$ \\
\hline 7 & 12 & $18.33 \pm 1.24$ & $x$ & $79.13 \pm 2.24$ & $x$ \\
\hline 10 & 11 & $19.64 \pm 0.73$ & $x$ & $63.90 \pm 1.10$ & $\mathrm{E}$ \\
\hline \multicolumn{2}{|c|}{ RI EXCB combined } & \multicolumn{2}{|c|}{$17.73 \pm 0.84$} & \multicolumn{2}{|c|}{$77.19 \pm 2.66$} \\
\hline RI CBXE 12 & 14 & $24.29 \pm 1.00$ & E & $73.73 \pm 1.64$ & $x$ \\
\hline 13 & 10 & $31.80 \pm 1.45$ & E & $68.13 \pm 1.88$ & $E$ \\
\hline 14 & 12 & $31.75 \pm 1.10$ & E & $64.77 \pm 1.68$ & $\mathrm{E}$ \\
\hline 15 & 13 & $18.69 \pm 1.51$ & $x$ & $76.33 \pm 0.95$ & $x$ \\
\hline 17 & 14 & $19.14 \pm 0.82$ & $x$ & $72.87 \pm 1.68$ & $x$ \\
\hline 18 & 13 & $29.46 \pm 1.51$ & $\mathrm{E}$ & $71.29 \pm 1.82$ & $x$ \\
\hline 20 & 10 & $18.80 \pm 0.71$ & $x$ & $72.00 \pm 1.35$ & $x$ \\
\hline 21 & 6 & $18.83 \pm 1.76$ & $x$ & $65.95 \pm 1.86$ & $E$ \\
\hline 22 & 4 & $19.50 \pm 0.96$ & $x$ & $73.40 \pm 1.30$ & $x$ \\
\hline 24 & 6 & $27.67 \pm 1.38$ & $\mathrm{E}$ & $77.90 \pm 1.33$ & $x$ \\
\hline 26 & 6 & $17.67 \pm 0.56$ & $x$ & $76.55 \pm 1.37$ & $x$ \\
\hline 27 & 6 & $33.33 \pm 1.02$ & $>\mathrm{E}$ & $64.45 \pm 1.86$ & $\mathrm{E}$ \\
\hline 28 & 6 & $23.33 \pm 0.67$ & $x$ & $61.25 \pm 1.47$ & $\mathrm{E}$ \\
\hline 29 & 6 & $18.67 \pm 1.38$ & $x$ & $75.18 \pm 1.86$ & $x$ \\
\hline 32 & 6 & $27.17 \pm 2.21$ & E & $78.82 \pm 1.89$ & $x$ \\
\hline 33 & 6 & $24.67 \pm 0.42$ & $x$ & $83.53 \pm 1.21$ & $x$ \\
\hline 34 & 6 & $32.67 \pm 1.73$ & $>E$ & $67.56 \pm 2.20$ & $E$ \\
\hline \multicolumn{2}{|c|}{ RI CBXE combined } & \multicolumn{2}{|c|}{$24.55 \pm 1.39^{\mathrm{d}}$} & \multicolumn{2}{|c|}{$72.39 \pm 1.42$} \\
\hline
\end{tabular}

"Sperm head width $\times 200 /$ sperm head length.

'CB or $E$, not significantly different from $C B A$ or $K E$ strain, respectively; $X$, intermediate, significantly different from both strains; $>E$ significantly higher proportion of abnormalities than in KE strain.

'Significantly different from $C B A(P<0.01)$.

'Significantly different from RI EXCB combined $(P<0.01)$.

difference was significant when the combined value (for fertilized and unfertilized ova) was calculated. Among recombinant inbred strains, two were CBA-like, three KE-like, one even more extreme than KE (RI EXCB 10) and three not different from either progenitor strain.

In one experiment, ova were examined during early stages of fertilization, with the aim of determining whether supplementary spermatozoa resulted from simultaneous penetration, or from inefficiency of the first spermatozoon to enter the vitellus. If the second answer was correct, more ova with supplementary spermatozoa would have been expected in the group showing less advanced stages of fertilization. Among 106 ova recovered from females killed $4-5 \mathrm{~h}$ after mating with KE males, only 41 were penetrated, i.e. the process of penetration was in progress. Each spermatozoon present in the perivitelline space of ova containing sperm head (or further stages of its transformation) in the vitellus was counted as supplementary; in the ova with a vitellus that was not yet penetrated, only the second spermatozoon was counted, if present. In the group of ova in very early stages of penetration (metaphase II or anaphase of meiosis), 13 of 23 contained supplementary spermatozoa in the perivitelline space, while in the group of ova in more advanced stages (second polar body extruded or already formed pronuclei) only 2 of 18 contained supplementary spermatozoa in the perivitelline space (test of independence with Yates' correction: chi square $8.25 ; P<0.01$ ). This finding suggests that the presence of supplementary spermatozoa may indicate inefficiency in penetrating the viteilus.

\section{Correlations between parameters of sperm morphology and sperm function}

For all tested parameters, correlation coefficients were calculated from mean values for each strain. There were significant correlations (Table 4) between some parameters related to sperm function: fertilization efficiency was correlated with 
Table 2. Sperm motility and Sperm Select penetration in progenitor strains CBA and KE, and in recombinant inbred strains RI EXCB and RI CBXE

\begin{tabular}{|c|c|c|c|c|c|c|c|}
\hline \multirow[b]{3}{*}{ Strain } & \multirow[b]{3}{*}{$n$} & & & \multicolumn{4}{|c|}{ Sperm Select penetration } \\
\hline & & \multicolumn{2}{|c|}{ Motile spermatozoa (\%) } & \multicolumn{2}{|c|}{$2.5 \mathrm{~cm}$ depth } & \multicolumn{2}{|c|}{ TVD $^{a}$} \\
\hline & & Mean \pm SEM & Type & Mean \pm SEM & Type & Mean \pm SEM & Type $^{b}$ \\
\hline CBA & 9 & $59.4 \pm 3.9^{\circ}$ & & $7.0 \pm 1.0^{c}$ & & $3.7 \pm 0.12^{\mathrm{c}}$ & \\
\hline $\mathrm{KE}$ & 8 & $43.2 \pm 1.5$ & & $1.4 \pm 0.5$ & & $2.7 \pm 0.14$ & \\
\hline RI EXCB 2 & 6 & $49.3 \pm 4.6$ & $E, C B$ & $3.5 \pm 1.6$ & $\mathrm{E}, \mathrm{CB}$ & $3.1 \pm 0.19$ & E \\
\hline 5 & 6 & $56.7 \pm 3.5$ & $\mathrm{CB}$ & $3.0 \pm 0.9$ & $\mathrm{E}$ & $3.0 \pm 0.18$ & E \\
\hline 6 & 6 & $51.0 \pm 4.1$ & E, CB & $2.0 \pm 0.9$ & E & $2.8 \pm 0.20$ & $E$ \\
\hline 10 & 6 & $57.3 \pm 2.4$ & $\mathrm{CB}$ & $8.5 \pm 2.0$ & $\mathrm{CB}$ & $4.3 \pm 0.25$ & $>\mathrm{CB}$ \\
\hline \multicolumn{2}{|c|}{ RI EXCB combined } & \multicolumn{2}{|c|}{$53.5 \pm 2.1$} & \multicolumn{2}{|c|}{$4.2 \pm 1.4$} & \multicolumn{2}{|c|}{$3.3 \pm 0.32$} \\
\hline RI CBXE 12 & 6 & $56.8 \pm 3.1$ & $\mathrm{CB}$ & $0.5 \pm 0.5$ & E & $2.0 \pm 0.22$ & $<\mathrm{E}$ \\
\hline 13 & 6 & $41.7 \pm 4.1$ & $E$ & $3.3 \pm 1.5$ & E & $3.4 \pm 0.35$ & $\mathrm{E}, \mathrm{CB}$ \\
\hline 15 & 6 & $37.0 \pm 7.3$ & E & $1.0 \pm 0.5$ & E & $2.8 \pm 0.25$ & $\mathrm{E}, \mathrm{CB}$ \\
\hline 17 & 7 & $55.1 \pm 6.2$ & $\mathrm{E}, \mathrm{CB}$ & $8.0 \pm 4.0$ & $\mathrm{E}, \mathrm{CB}$ & $3.4 \pm 0.39$ & CB \\
\hline 20 & 6 & $54.3 \pm 1.7$ & $\mathrm{CB}$ & $6.0 \pm 1.6$ & $\mathrm{CB}$ & $3.6 \pm 0.31$ & $\mathrm{CB}$ \\
\hline \multicolumn{2}{|c|}{ RI CBXE combined } & \multicolumn{2}{|c|}{$49.0 \pm 4.0$} & \multicolumn{2}{|c|}{$3.8 \pm 1.4$} & \multicolumn{2}{|c|}{$3.0 \pm 0.30$} \\
\hline
\end{tabular}

${ }^{3}$ Theoretical Vanguard Distance; the point at which regression lines (c.f. Fig. 3) bisected the abscissa.

${ }^{b} \mathrm{CB}$ or $\mathrm{E}$, not significantly different from $\mathrm{CBA}$ or $\mathrm{KE}$ strain, respectively.

'Significantly different from KE $(P<0.01)$.

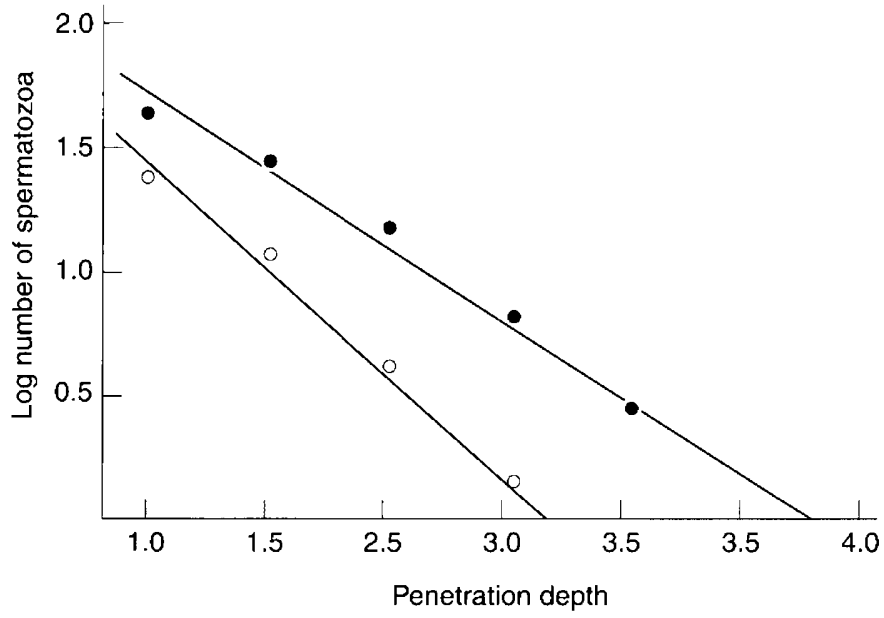

Fig. 3. Regression of the number of spermatozoa (in log transformation) that penetrated Sperm Select on the depth of penetration in two strains of mice: $\mathrm{KE}(O)$ and $\mathrm{CBA}(\bullet)$. The point at which the regression line crosses the abscissa represents the theoretical vanguard distance (TVD). Data points indicate the mean values obtained from eight (KE) and nine (CBA) male mice.

Sperm Select penetration measured at a depth of $2.0 \mathrm{~cm}$ and $2.5 \mathrm{~cm}$ and the proportion of motile spermatozoa was correlated with Sperm Select penetration at 1.0 and $1.5 \mathrm{~cm}$ depth. None of these parameters was significantly correlated with the proportion of abnormal spermatozoa, although for motility and Sperm Select penetration at a depth of 1.0 and $1.5 \mathrm{~cm}$, and for supplementary spermatozoa in all ova, correlation coefficients approached the level of significance $(P \leq 0.10)$. Thus strains with high incidence of sperm abnormalities showed some tendency to be less efficient in motility and Sperm Select penetration, and to yield more supplementary spermatozoa in fertilized and unfertilized eggs.

However, there was a highly significant negative correlation between the index of sperm head shape and the proportion of sperm abnormalities, both for 11 strains used for testing sperm function (Table 4) and for 26 strains, including incipient 


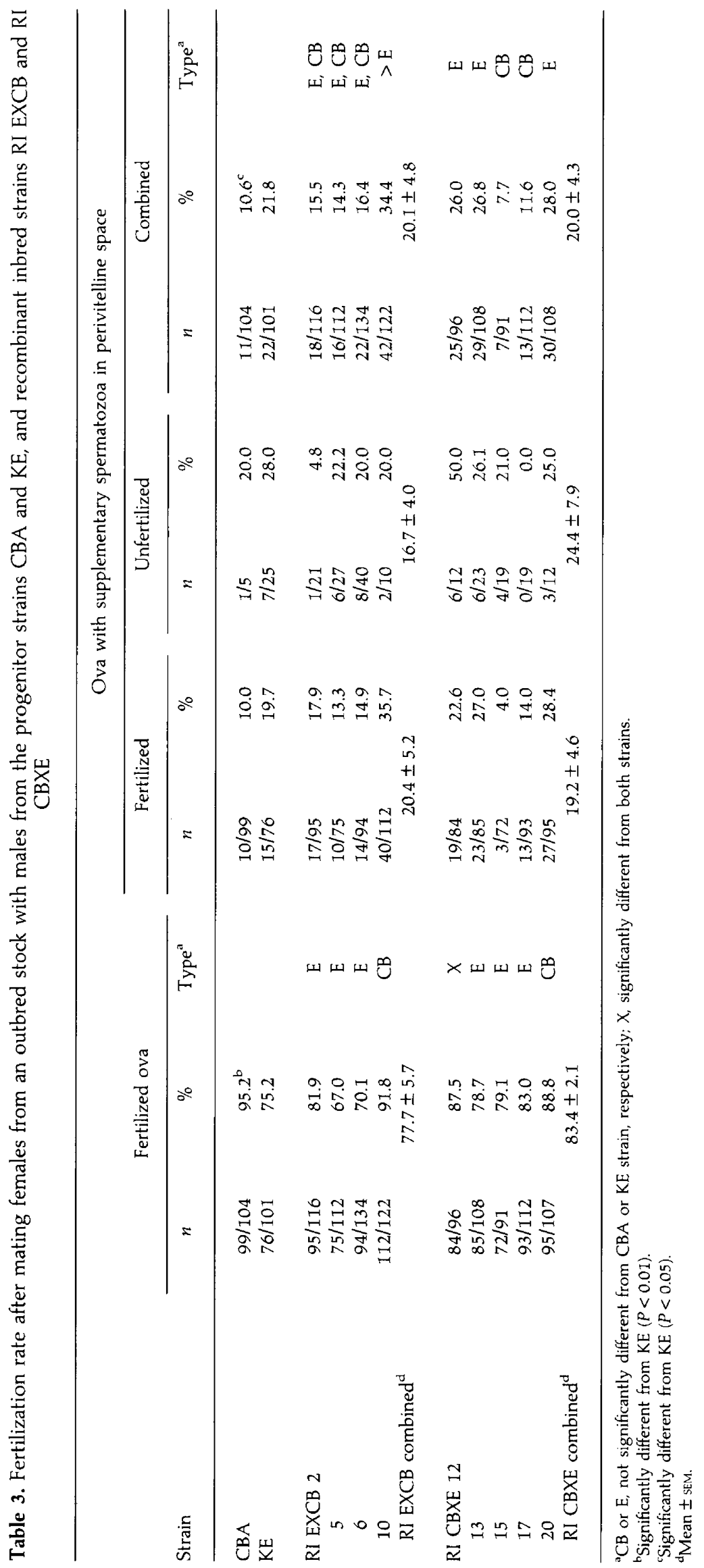


Table 4. Correlation coefficients $(r)$ between parameters of sperm quality (calculated from mean values for recombinant inbred and parental strains; $n=11$; d.f. $=9$ )

\begin{tabular}{|c|c|c|c|c|c|c|c|c|}
\hline \multirow[b]{2}{*}{ Sperm parameters } & \multicolumn{4}{|c|}{ Sperm Select penetration depth ${ }^{a}$} & \multirow{2}{*}{$\begin{array}{c}\text { Abnormal } \\
\text { spermatozoa }\end{array}$} & \multirow[b]{2}{*}{ Head shape } & \multirow[b]{2}{*}{ Motility } & \multirow[b]{2}{*}{ Fertilization } \\
\hline & $1.0 \mathrm{~cm}$ & $1.5 \mathrm{~cm}$ & $2.0 \mathrm{~cm}$ & $2.5 \mathrm{~cm}$ & & & & \\
\hline Motility (\%) & $0.65^{*}$ & $0.61 *$ & 0.57 & 0.53 & -0.55 & 0.28 & & \\
\hline Fertilization (\%) & 0.38 & 0.58 & $0.66^{*}$ & $0.61^{*}$ & -0.26 & 0.15 & 0.40 & \\
\hline Supplementary spermatozoa in fertilized ova (\%) & 0.26 & 0.27 & 0.10 & 0.33 & 0.45 & $-0.68^{*}$ & 0.23 & 0.29 \\
\hline Supplementary spermatozoa in all ova $(\%)$ & 0.08 & 0.07 & -0.06 & 0.17 & 0.52 & $-0.72^{* *}$ & 0.16 & 0.25 \\
\hline Head shape (index) & 0.25 & 0.23 & 0.28 & -0.02 & $-0.71^{* *}$ & & & \\
\hline Abnormal spermatozoa (\%) & -0.51 & -0.53 & -0.49 & -0.39 & & & & \\
\hline
\end{tabular}

${ }^{2}$ For $3.0 \mathrm{~cm}$ depth and for theoretical vanguard distance (TVD) values correlation coefficients were not significant

Significant correlation $\left({ }^{*} P<0.05 ; * * P<0.01\right)$.

recombinant inbred strains $(r=-0.498$; d.f. $=24 ; P<0.01)$. Moreover, the index of sperm head shape was negatively correlated with the incidence of supplementary spermatozoa in the perivitelline space of fertilized ova, as well as of all ova. Thus recombinant inbred strains possessing sperm heads similar to those in the CBA strain tended to show a lower proportion of abnormalities and of supplementary spermatozoa.

\section{Discussion}

The two progenitor strains, KE and CBA, differ significantly in several aspects of sperm morphology and function. Thus, the recombinant inbred strains developed from such contrasting strains seemed to be good models for testing the factors influencing sperm quality and relationships between different parameters.

It was important to find adequate criteria for testing functional competence of spermatozoa. The ability to fertilize eggs is strongly dependent on the quality of both eggs and spermatozoa, for example in matings with CBA females, both CBA and KE males fertilize $100 \%$ of eggs, while in matings with KE females or with females from our outbred stock, the performance of KE males is significantly poorer (Krzanowska, 1970). Therefore outbred females were used in this study to test fertilization efficiency of males from recombinant inbred strains. Motility and the hyaluronic acid penetration (Sperm Select) test were included as objective measures of sperm performance, that do not involve the quality of eggs. The Sperm Select test that gives an objective measure of functional competence of human spermatozoa (Aitken et al., 1992) also appeared suitable for mouse spermatozoa, as shown by positive correlations with fertilization efficiency. Most reliable in this respect were values of the number of spermatozoa that penetrated 2.0 and $2.5 \mathrm{~cm}$ depth of Sperm Select. They were not dependent on the percentage of motile spermatozoa; this last mentioned parameter showed a correlation with the number of spermatozoa at lower depths of Sperm Select (1.0 and $1.5 \mathrm{~cm}$ ), and was not correlated with fertilization efficiency.

In the progenitor strains, $\mathrm{KE}$ and $\mathrm{CBA}$, high indices of tests measuring functional competence of spermatozoa were linked with a low proportion of sperm abnormalities, and vice versa. Although abnormal spermatozoa cannot be directly responsible for fertilization failure because they are largely prevented from crossing the utero-tubal junction (Krzanowska, 1974) and from penetrating the egg investments (Krzanowska and Lorenc, 1983), their presence could indicate that the process of spermatogenesis was affected, so that some spermatozoa of normal appearance may be functionally inefficient. Among the recombinant inbred strains, correlations between sperm normality and the indices of fertilization efficiency were not significant. This may be due to the fact that the incidence of abnormalities was not very severe even in the most affected strains; in dogs, the percentage of spermatozoa with normal morphology below which fertility was adversely affected was found to be $60 \%$ (Oettle, 1993), while in our strains the highest proportion of abnormal sperm heads did not surpass $30 \%$. However, there was a tendency (correlation coefficients approaching significance) for the strains with a higher incidence of abnormalities to show lower sperm motility, less efficient penetration into Sperm Select and a higher proportion of supplementary spermatozoa in the perivitelline space. The meaning of the last mentioned parameter deserves discussion.

The fact that the proportion of supplementary spermatozoa differs between inbred strains of mice, and is largely influenced by the strain of the male was first stated by Braden (1958). He discussed two possible explanations for the higher incidence of supplementary spermatozoa in eggs fertilized by $\mathrm{C} 57$ males: first, a higher sperm interception rate as a consequence of greater motility of $\mathrm{C} 57$ spermatozoa or their greater number at the site of fertilization and second, the delay between the penetration of the zona and the attachment of the sperm head to the vitellus, with the result that a greater proportion of eggs is penetrated by an extra spermatozoon before the zona reaction begins. The results of diallele crosses between four mouse strains (Krzanowska, 1970), showing a correlation between the proportion of unfertilized ova (half of them with penetrated zona) and those containing supplementary spermatozoa, suggest that the penetration of the egg by more than one spermatozoon may also be caused by the inability of some spermatozoa from certain strains to enter the vitellus. The present results confirmed this view in relation to eggs fertilized by KE males: a higher incidence of supplementary spermatozoa 
in the eggs that were less advanced in the process of fertilization may indicate that the first spermatozoon penetrating the zona could not enter the vitellus, so that the egg had to wait for the second more successful one, or remained unfertilized. Thus, at least when KE and CBA strains are compared, a higher incidence of supplementary spermatozoa is assumed to indicate their inefficiency. In this connection it should be noted that studies using an in vitro fertilization assay, in which human spermatozoa were fused with hamster oocytes, have demonstrated that a large proportion of such cases involve the failure of acrosome-reacted spermatozoa to fuse with the vitelline membrane of the oocyte (Aitken, 1992).

The lack of correlation between the incidence of supplementary spermatozoa and fertilization efficiency could be explained by independent segregation of sperm quality factors in recombinant inbred strains; for example more spermatozoa at the site of fertilization, or their higher motility, could facilitate the penetration of the egg by the second spermatozoon, thus compensating for the failure of the first inefficient spermatozoon. The recombinant inbred strain EXCB 10 seems to be a good example of such interactions, having the highest incidence of supplementary spermatozoa (linked with very low index of sperm head shape), but showing the highest scores of motility, penetration into Sperm Select, and fertilization.

There was a highly significant negative correlation between the index of sperm head shape and both the proportion of abnormalities and the incidence of supplementary spermatozoa under the zona pellucida of fertilized and unfertilized eggs. Two interpretations are possible: either these features are genetically linked or KE-like sperm heads, with a lower width to length ratio, are more prone to undergo deformations during the process of spermiogenesis and are less efficient in establishing contact with the vitellus after they have penetrated the zona pellucida. Although the mechanism of entry of the spermatozoa into the vitellus is not yet fully known, it was found (Blobel et al., 1992) that the mammalian spermatozoon contains a unique membrane recognition/fusion complex (PH30) involved in sperm-oocyte fusion. It is possible that sperm head shape may influence the localization or proper functioning of this complex.

Human spermatozoa with heads showing a higher ratio of the length to width also tended to show a higher proportion of abnormalities, lower rate of fertilization and of binding to the zona pellucida (Liu and Baker, 1992). The fact that similar relationships were observed both for human and mouse spermatozoa, which are so different in shape, suggests that sperm width may be important for the success of egg penetration.

As expected, all investigated characters were polygenic, showing the involvement of at least two genes. When comparing two groups of recombinant inbred strains (EXCB and CBXE) developed from reciprocal crosses of progenitor strains, the beneficial effect of the CBA-derived $Y$ chromosome was stated only with respect to sperm normality but not to head shape. This confirms earlier findings (Krzanowska, 1969, 1976; Styrna et al., 1991) about the important role of the $Y$ chromosome in determining the level of sperm abnormalities. A multiple-copy spermiogenesis gene was found in the long arm of this chromosome (Conway et al., 1994). Comparison of two consomic strains, KE and KE-YCBA, with different $Y$ chromosomes on the same genetic background, showed the favourable effect of the CBA-derived $Y$ chromosome on both sperm normality and fertilization efficiency (Krzanowska, 1969, 1986). In contrast, among the recombinant inbred strains, this effect was not observed in relation to sperm function. It seems that $Y$ chromosome-linked defects of spermiogenesis (leading to production of abnormal spermatozoa) could be compensated in some recombinant inbred strains by segregating CBA-derived autosomal genes, important both for sperm morphology and function.

The authors thank D. Buda-Lewandowska for preparing photomicrographs needed for sperm head measurements. This work was supported by the Polish Scientific Research Committee (KBN) within the Project 418109101.

\section{References}

Aitken J (1992) A family of fusion proteins Nature 356 196-197

Aitken RJ, Bowie H, Buckingham D, Harkiss D, Richardson DW and West KM (1992) Sperm penetration into a hyaluronic acid polymer as a means of monitoring functional competence Journal of Andrology 13 44-54

Bailey DW (1971) Recombinant inbred strains Transplantation 11 325-327

Bailey DW (1981) Recombinant inbred strains and bilineal congenic strains. In The Mouse in Biomedical Research Vol. I pp 223-239 Eds HL Foster, JD Small and JG Fox. Academic Press, New York

Blobel CP, Wolfsberg TG, Turck CW, Myles DG, Primakoff $P$ and White JM (1992) A potential fusion peptide and an integrin ligand domain in a protein active in sperm-egg fusion Nature 356 248-252

Braden AWH (1958) Variation between strains in the incidence of various abnormalities of egg maturation and fertilization in the mouse Journal of Genetics 56 37-47

Conway SJ, Mahadevaiah SK, Darling SM, Capel B, Rattigan AM and Burgoyne PS (1994) Y353/B: a candidate multiple-copy spermiogenesis gene on the mouse $Y$ chromosome Mammalian Genome 5 203-210

Kaleta $E$ (1977) Influence of genetic factors on the fertilization of mouse ova in vitro Journal of Reproduction and Fertility 51 375-381

Kaskar K, Franken DR, van der Horst G, Oehninger S, Kruger TF and Hodgen GD (1994) The relationship between morphology, motility and zona pellucida binding potential of human spermatozoa Andrologia 26 1-4

Krzanowska H (1969) Factor responsible for sperm head abnormality located on the $Y$ chromosome in mice Genetical Research (Cambridge) 13 17-24

Krzanowska H (1970) Relation between fertilization rate and penetration of eggs by supplementary spermatozoa in different mouse strains and crosses Journal of Reproduction and Fertility 22 199-204

Krzanowska H (1974) The passage of abnormal spermatozoa through the utero-tubal junction of the mouse Journal of Reproduction and Ferrility $\mathbf{3 8}$ 81-90

Krzanowska H (1976) Inheritance of sperm head abnormality types in mice the role of the Y chromosome Genetical Research (Cambridge) 28 189--198

Krzanowska H (1986) Interstrain competition amongst mouse spermatozoa inseminated in various proportions, as affected by the genotype of the $Y$ chromosome Journal of Reproduction and Fertility 77 265-270

Krzanowska $\mathrm{H}$ and Lorenc $\mathrm{E}$ (1983) Influence of egg investments on in vitro penetration of mouse eggs by misshapen spermatozoa Journal of Reproduction and Fertility 68 57-62

Liu DY and Baker HWG (1992) Morphology of spermatozoa bound to the zona pellucida of human oocytes that failed to fertilize in vitro journal of Reproduction and Fertility 94 71-84

Oettle EE (1993) Sperm morphology and fertility in the dog Journal of Reproduction and Fertility Supplement $47257-260$

Snedecor GW (1955) Statistical Methods pp 485 Iowa State College Press, Ames

Styrna J, Klag J and Moriwaki K (1991) Influence of partial deletion of the $Y$ chromosome on mouse sperm phenotype Journal of Reproduction and Fertility 92 187-195 